\title{
A POINTWISE ERGODIC THEOREM FOR THE GROUP OF RATIONAL ROTATIONS ${ }^{1,2}$ \\ BY
}

\author{
LESTER E. DUBINS AND JIM PITMAN
}

ABSTRACT. Let $f$ be a bounded, measurable function defined on the multiplicative group $\Omega$ of complex numbers of absolute value 1 , and define

$$
f_{n}(\omega)=\frac{1}{n} \sum_{i=1}^{n} f\left(z_{n}^{i} \omega\right), \quad \omega \in \Omega,
$$

where $z_{n}$ is a primitive $n$th root of unity. The present paper generalizes this result of Jessen [1934]: if $n(k)$ is an increasing sequence of positive integers with $n(k)$ dividing $n\left(k^{\prime}\right)$ whenever $k<k^{\prime}$, then $f_{n(k)}$ converges almost surely as $k \rightarrow \infty$.

1. Introduction. For $f$ a real-valued function defined on the unit circle $\Omega$, let

$$
f_{n}(\omega)=\frac{1}{n} \sum_{i=1}^{n} f\left(z_{n}^{i} \omega\right)
$$

where $\omega \in \Omega$ and $z_{n}^{i}$ is the $i$ th power of $z_{n}$, a primitive $n$th root of unity.

Jessen [1934] raised, but did not answer, the question whether, for integrable $f, f_{n}$ converges almost surely. He did show that $f_{n}$ converges almost surely provided $n$ ranges only over the elements of a chain, that is, an infinite subset $K$ of the positive integers, $N$, with the property that each element of $K$ is a divisor of the next. Jessen's question was not answered until Rudin [1964] exhibited a bounded, measurable function $f$ for which $f_{n}$ diverges everywhere.

For each set $\mathcal{Q}$ of functions $f$, a subset $K$ of $N$ is an $\mathcal{Q}$-set if, for every $f \in \mathbb{Q}$, for $k$ restricted to $K, f_{k}$ converges a.s. as $k \rightarrow \infty$. For $k \in N, l \in N$, let $k \vee l$ be the least common multiple of $k$ and $l$ and, for $K \subset N, L \subset N$, let $K \vee L$ be the set of all $k \vee l$ for $k \in K, l \in L$. Let $\mathfrak{T}$ be the set of bounded, measurable $f$. Baker [1976] discovered that if $K$ and $L$ are $\Re$-sets, then so is $K \vee L$. The dimension of a nonempty set $K$ of positive integers is the least positive integer $d$ such that there are chains $K_{1}, \ldots, K_{d}$ for which $K$ is a subset of $K_{1} \vee \cdots \vee K_{d}$. As is an immediate consequence of Jessen's

Received by the editors April 28, 1978 and, in revised form, July 20, 1978.

AMS (MOS) subject classifications (1970). Primary 60G45, 22D12, 28 A65.

Key words and phrases. Martingales, ergodic theory, permutable groups, conditional independence.

${ }^{1}$ The research of the two authors was supported by National Science Foundation Grants MCS77-01665 and MCS75-10376-A01, respectively.

${ }^{2}$ We thank Marek Kanter for calling important references to our attention. 
and Baker's results together, every set $K$ of finite dimension is an $\mathfrak{N}$-set. More is true, namely, every such $K$ is an $\mathcal{L}_{p}$-set for every $p>1$, which is a principal purpose of the present paper to demonstrate. Somewhat more sharply:

THEOREM 1.1. For each positive integer $d$, if $K$ has dimension $d$, then $K$ is an

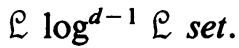

As usual, $\mathcal{E} \log ^{d-1} \mathcal{E}$ is the set of all $f$ such that $\phi_{d} \circ f$ is integrable, where $\phi_{d}: R \rightarrow R^{+}$is defined by $\phi_{d}(x)=|x|(\log |x|)^{d-1}$ if $|x|$ exceeds 1 and is zero otherwise.

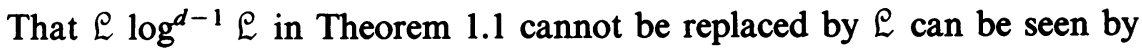
consideration of the set $K$ of dimension 2 which consists of the integers of the form $2^{i} 3^{j}$ (Baker [1976]). We presume that for no $d>2$ can $\mathcal{L} \log ^{d-1} \mathcal{L}$ be

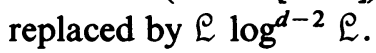

A set $K$ of positive integers has breadth at most $d$ if the least common multiple of every finite subset of $K$ is the least common multiple of at most $d$ elements of that subset; the least such $d$ is the breadth of $K$ and, if no such $d$ exists, $K$ has infinite breadth. Let $\mathscr{B}$ and $\mathscr{D}$ be the collection of $K$ of finite breadth and of finite dimension, respectively, and let $\mathcal{C}$ be the collection of $K$ which are $\Re$-sets (' $C$ ' for 'convergence'). Then $\mathscr{B} \supseteq \mathcal{C} \supseteq \mathbb{D}$, where the first inclusion was established by Rudin [1964], and the second, as noted above, follows from Jessen's and Baker's results together. An example provided in \$3 shows that the inclusion $\mathscr{B} \supset \mathscr{D}$ is strict, but we leave open the interesting question whether $\mathcal{C}$ is identical with $\mathscr{B}$, or with $\mathscr{D}$, or with neither.

A generalization of Theorem 1.1 to groups which are not necessarily commutative is offered in $\$ 4$, and an application is then made to a law of large numbers for multiparameter, semiexchangeable processes.

2. Proof of Theorem 1.1. For $f \in \mathcal{L}$, let $f_{n}$ be as in (1.1) and define $E_{n}$ by $E_{n} f=f_{n}$. Give Borel subsets of $\Omega$ the usual uniform probability measure. Then $E_{n}$ acts as a conditional expectation operation given $\mathscr{F}_{n}$, the $\sigma$-field of Borel sets $F$ such that $\omega \in F$ implies $z_{n}^{i} \omega \in F$ for all $1<i<n$. As is easily verified,

$$
E_{m} E_{n}=E_{n} E_{m}=E_{m \vee n}, \quad m, n \in N,
$$

which is an expression of the fact that the $\sigma$-fields $\mathscr{F}_{m}$ and $\mathscr{F}_{n}$ are conditionally independent given $\mathscr{F}_{m} \cap \mathscr{F}_{n}$ (see II.T.45 of Dellacherie and Meyer [1975]).

Suppose that $K$ equals $K_{1} \vee \cdots \vee K_{d}$ where each $K_{r}$ is a chain. Let $K_{r}(n)$ be the $n$th element of $K_{r}$ and, for $i \in N^{d}$, let $k(i)$ be the least common multiple of the $d$ integers $K_{r}(i(r)), 1<r<d$. For $i \in N^{d}$, set $E_{i}$ equal to $E_{k(i)}$. Verify that the $E_{i}, i \in N^{d}$, are conditional expectation operators which satisfy 


$$
E_{i} E_{j}=E_{i \vee j}, \quad i \in N^{d}, j \in N^{d},
$$

where $i \vee j \in N^{d}$ is the supremum of $i$ and $j$ in the coordinatewise ordering of $N^{d}$.

Condition (2.1) already implies that for $f \in \mathcal{L} \log ^{d-1} \mathcal{L}, E_{i} f$ converges almost surely to a limit $E_{\infty}(f)$. This means that, setting a null set aside, for each $\varepsilon>0$, there is an integer $l$ such that for all $i \in N^{d}$ each of whose coordinates exceeds $l,\left|E_{i} f-E_{\infty} f\right|<\varepsilon$. But the $E_{i} f$ converge in a stronger sense.

A countable array $e_{i}$ of real numbers converges rearrangeably to a real number $e_{\infty}$ if, for some (and hence every) sequential ordering of the $e_{i}$, the sequence converges to $e_{\infty}$.

Let $i(r, n)$ be that element of $N^{d}$ which has all of its coordinates equal to 1 , except the $r$ th, which equals $n$. For each $r$, the set of all $i(r, n)$ as $n$ ranges over the positive integers is the $r$ th axis. Let $E_{r, \infty}$ be the infimum of $E_{i}$ as $i$ ranges over the $r$ th axis. Here, the infimum of a collection of conditional expectation operators is the conditional expectation operator given the intersection of the corresponding $\sigma$-fields.

As is well known and easily verified, an $\mathfrak{L}_{1}$-function which is invariant under a dense set of rotations is constant almost certainly. (A formal argument may be seen in [Dubins, 1977, Lemma 2].) Therefore,

$$
\begin{aligned}
& \text { for each } r, 1 \leqslant r \leqslant d \text {, and each } f \in \mathcal{L}_{1}, \\
& E_{r, \infty} f \text { is a constant almost surely. }
\end{aligned}
$$

As will soon be seen, (2.1) and (2.2) together imply that for each $f \in$ $\mathcal{L} \log ^{d-1} \mathfrak{L}$, there is a null set outside of which $E_{i} f$ converges rearrangeably to the constant $E f$.

Verify that as $i$ ranges over $N^{d}, k(i)$ ranges over all of $K$, so the range of the array $\left(E_{i} f, i \in N^{d}\right)$ is identical with the range of $\left(f_{k}, k \in K\right)$. Since the $E_{i} f$ converge rearrangeably almost surely, $f_{k}$ converges (in the usual sense) almost surely as $k$ ranges over $K$. Since there plainly was no real loss in generality in assuming that $K$ was equal to $K_{1} \vee \cdots \vee K_{d}$ rather than a subset of it, Theorem 1.1 is fully proved modulo only the proof of this assertion:

THEOREM 2.1. Let $\left(E_{i}, i \in N^{d}\right)$ be an array of conditional expectation operators which satisfy (2.1) and (2.2). Then for any $f \in \mathcal{L} \log ^{d-1} \mathcal{L}$, there is a null set outside of which $E_{i} f$ converges rearrangeably to the constant $E f$.

For Theorem 2.1, the underlying countably additive probability space can be arbitrary. An instance of Theorem 2.1 was established by Smythe [1973] following Cairoli [1970]. Priority goes also to Cairoli and Walsh [1975], Smythe [1974] and Gut [1976]. Indeed, as shown by Gut [1976] an inequality 
of Cairoli can be used to show that for $f \in \mathcal{L} \log ^{d-1} \mathcal{L}$, condition (2.1) is sufficient for the almost sure convergence of $E_{i} f$, in the coordinatewise ordering. Moreover, by an argument of Smythe [1973], Theorem 2.1 can be derived from Gut's convergence theorem. It is simpler, however, to derive both theorems directly from the maximal inequality of Cairoli [1970] which, slightly reformulated, states:

There are constants $a$ and $b$ which depend on $d$ only such that, if $\left(E_{i}, i \in N^{d}\right)$ satisfies (2.1) then, for $\varepsilon, \delta>0$,

$$
P\left(\sup _{i}\left|E_{i} f\right| \geqslant \delta\right) \leqslant \varepsilon\left(1+a E \phi_{d}(c f)\right)
$$

where $c=b / \varepsilon \delta$.

Let $E_{\infty}$ be the infimum of the conditional expectation operators ( $E_{i}$, $i \in N^{d}$ ).

LEMMA 2.1. Suppose $\left(E_{i}, i \in N\right)$ satisfies (2.1), $f \in \mathcal{L} \log ^{d-1} \mathcal{L}, E_{\infty} f=0, j$ : $N \rightarrow N^{d}$ is increasing and the infimum of $E_{j(n)}(n \in N)$ is $E_{\infty}$. Then $\sup _{i}\left|f_{j(n)+i}\right|$ $\rightarrow 0$ almost surely as $n \rightarrow \infty$.

Proof. Because $\sup _{i}\left|f_{j(n)+i}\right|$, say $f_{n}^{*}$, is decreasing in $n$, it suffices to show that $P\left(f_{n}^{*} \geqslant \delta\right) \rightarrow 0$ as $n \rightarrow \infty$. Apply (2.3) with $\left(f_{j(n)}, E_{j(n)+i}\right)$ substituted for $\left(f, E_{i}\right)$ to see that it is enough to show: for each $c>0, E \phi\left(c f_{j(n)}\right) \rightarrow 0$ as $n \rightarrow \infty$. Fix $c$, put $g_{n}=c f_{j(n)}$ and check that $\left(g_{n}, E_{j(n)} ; n \in N\right)$ is a onedimensional reversed martingale which converges almost surely to $c f_{\infty}=0$ as $n \rightarrow \infty$. Since $\phi_{d}(x)=0$ for $|x| \leqslant 1, \phi_{d} \circ g_{n} \rightarrow 0$ almost surely, too. Since $\phi_{d}$ is convex, $\phi_{d} \circ g_{n}$ is a nonnegative, reversed submartingale. Therefore, by [13, Corollary V-3-13],

$$
\lim _{n} E \phi_{d} \circ g_{n}=E \lim _{n} \phi_{d} \circ g_{n}=0,
$$

which completes the proof.

To obtain Theorem 2.1, first reduce to the case $E f=0$ and apply Lemma 2.1 with $j(n)=j_{r}(n)$ for each $r \in D$. To obtain Gut's theorem, reduce to the case $E_{\infty} f=0$ and apply Lemma 2.1 , with $j(n)=(n, \ldots, n)$.

3a. A divergent, two-parameter, bounded, reversed martingale. Let $p_{\boldsymbol{n}}$ be the $n$th prime, $\Pi_{n}$ the product of the first $n$ primes, and let $\Pi_{0}$ be 1 . Let $i=\left(i_{1}, i_{2}\right)$ range over $N^{2}$ and define $k(i)$ thus.

$$
k(i)= \begin{cases}\Pi_{m(m+1)} & \text { if } i_{1}+i_{2}=2 m, \\ \Pi_{m(m+1)} p_{m(m+1)+i_{1}+1} & \text { if } i_{1}+i_{2}=2 m+1\end{cases}
$$

Since $k(i)$ divides $k(j)$ whenever $i$ is less than $j$ in the coordinatewise ordering, $\left(f_{k(i)}, \mathscr{F}_{k(i)} ; i \in N^{2}\right)$ is a reversed martingale for any $f \in \mathcal{E}$, where $f_{n}$ is defined by (1.1). (For $E_{i}=E_{k(i)}$, (2.1) fails, though $E_{i} E_{j}=E_{j} E_{i}$ and (2.2) holds.) As is easily verified, the range $K$ of $\left(k(i), i \in N^{2}\right)$ has infinite breadth, 
so by the construction of Rudin [1964] there exists a bounded Borel function $f$ such that $f_{k}$ diverges everywhere as $k \rightarrow \infty$ through $K$. The array $\left(f_{k(i)}, i \in\right.$ $N^{2}$ ) is a.s. divergent, for it fails to converge rearrangeably anywhere, though each row sequence $f_{k\left(i_{1},\right)}$ converges to $E f$ a.s. by Jessen's theorem, as does each column sequence.

This example supplements the example of a divergent, directed martingale given by Dieudonne [1950]. Another, and much simpler example than either of these, will be offered in a forthcoming note.

3b. A set of integers of finite breadth and of infinite dimension. Let $n$ be a positive integer, let $p_{n}$ be the $n$th prime, let $K_{n}$ be the set of all integers $k$ such that for some $m \leqslant n, k$ is the product of all, except at most one, of the first $m$ primes, $2=p_{1}<p_{2}<\cdots<p_{m}$, and let $K=\cup_{n} K_{n}$. Then $K$ is the desired example. That $K$ has breadth 2 is obvious. What must be demonstrated, therefore, is that it has infinite dimension. For this, it plainly suffices to show that the dimension of $K_{n}$ converges to $\infty$ as $n \rightarrow \infty$.

A $d$-scheme is a $d$-tuple $\sigma=\left(\sigma_{1}, \ldots, \sigma_{d}\right)$ where each $\sigma_{r}$ is a prime-valued function defined on a finite set $D_{r}$ of integers. For each $j \in D_{r}$ let $\hat{\sigma}_{r}(j)$ be the product over all $i \in D_{r}$ which do not exceed $j$ of $\sigma_{r}(i)$, and let $R(\sigma)$, the reach of $\sigma$, be the smallest set of integers which satisfies these two conditions: (a) for each $r, 1 \leqslant r \leqslant d$, and each $j \in D_{r}, \hat{\sigma}_{r}(j) \in R(\sigma)$; (b) the least common multiple of each finite subset of $R(\sigma)$ is an element of $R(\sigma)$.

To show that the dimension of a finite set of positive integers exceeds $d$, it plainly suffices to show that the set is not included in $R(\sigma)$ for any $d$-scheme $\sigma$.

For a $d$-scheme $\sigma^{\prime}=\left(\sigma_{1}^{\prime}, \ldots, \sigma_{d}^{\prime}\right)$, define the relation $\sigma^{\prime} \leqslant \sigma$ if, for each $r$, $1 \leqslant r \leqslant d, \sigma_{r}^{\prime}$ is the restriction of $\sigma_{r}$ to a subset $D_{r}^{\prime}$ of $D_{r}$.

Call $\sigma$ minimal for $K_{n}$ if these two conditions hold: (a) $K_{n} \subset R(\sigma)$; and (b) if $\sigma^{\prime} \leqslant \sigma$ and $K_{n} \subset R\left(\sigma^{\prime}\right)$, then $\sigma^{\prime}=\sigma$. Call $\sigma$ orderly if, for each $r, 1 \leqslant r \leqslant$ $d$, and each $i$ and $j$ in $D_{r}$ with $i<j, \sigma_{r}(i)<\sigma_{r}(j)$.

LEMMA 3.1. Any scheme which is minimal for $K_{n}$ is orderly.

Proof. Let $\sigma$ be a $d$-scheme which is minimal for $K_{n}$. Trivially, for all $r$, $1 \leqslant r \leqslant d$, and all $j \in D_{r}, \sigma_{r}(j) \leqslant p_{n}$. Moreover, as is easily verified, the inequality is strict unless $j$ is the largest element of $D_{r}$. Let $\sigma_{r}^{\prime}$ be the restriction of $\sigma_{r}$ to the set of $j \in D_{r}$ for which $\sigma_{r}(j)<p_{n}$. Plainly, $K_{n-1} \subset R\left(\sigma^{\prime}\right)$. In fact, $\sigma^{\prime}$ is minimal for $K_{n-1}$. For otherwise, as will soon be shown, there exists a $d$-scheme $\rho \leqslant \sigma, \rho \neq \sigma$ with these two properties: (a) $K_{n-1} \subset R(\rho)$, and (b) for each $k \in K_{n}, k \notin K_{n-1}, \exists k^{*} \in R(\rho)$ such that $p_{n}$ divides $k^{*}$, and $k^{*}$ divides $k$. For such a $k, k^{\prime}=k / p_{n}$ is plainly in $K_{n-1}$ and hence $k^{\prime} \in R(\rho)$. So $k$, the l.c.m. of two elements of $R(\rho)$, is itself an element of $R(\rho)$. That is, $K_{n} \subset R(\rho)$, which contradicts the assumption that $\sigma$ is minimal for $K_{n}$. 
Return to the proof that the asserted $\rho$ does exist if $\sigma^{\prime}$ were not minimal for $K_{n-1}$. For let $\rho^{\prime}$ be a $d$-scheme which satisfies $\rho^{\prime} \leqslant \sigma^{\prime}, \rho^{\prime} \neq \sigma^{\prime}$ and $K_{n-1} \subset$ $R\left(\rho^{\prime}\right)$, let $\rho^{\prime \prime}$ be defined by letting $\rho_{r}^{\prime \prime}$ be the restriction of $\sigma_{r}$ to the set of $j \in D_{r}$ for which $\sigma_{r}(j)=p_{n}$, and let $\rho_{r}(j)$ be $\rho_{r}^{\prime}(j)$ or $\rho_{r}^{\prime \prime}(j)$ according as $j$ is in the domain of $\rho_{r}^{\prime}$ or of $\rho_{r}^{\prime \prime}$. That $\rho$ has the desired properties is verified without difficulty, so the proof that $\sigma^{\prime}$ is minimal for $K_{n-1}$ is complete. So not only is $\sigma_{r}(j)<p_{n}$ unless $j$ is the largest element of $D_{r}$, but also $\sigma_{r}^{\prime}(j)<p_{n-1}$ unless $j$ is the largest element in the domain of $\sigma_{r}^{\prime}$. A simple induction now completes the proof that $\sigma$ is orderly.

Associate to each $d$-scheme $\sigma$ and prime $p$ the set $S_{p}$ of all $r$ such that for some $j, \sigma_{r}(j)=p$.

LEMMA 3.2. Suppose that $\sigma$ is an orderly scheme, $p$ and $q$ are primes, $p<q$, $S_{p} \supseteq S_{q}, k \in R(\sigma)$ and $q$ divides $k$. Then $p$ divides $k$.

Proof. Easy and omitted.

LEMMA 3.3. Let $\sigma$ be an orderly $d$-scheme for which $K_{n} \subset R(\sigma)$, and suppose that $p$ and $q$ are primes with $2 \leqslant p<q \leqslant p_{n}$. Then $S_{p}$ does not include $S_{q}$. In particular, $S_{p}$ and $S_{q}$ are distinct, so $n \leqslant 2^{d}-1$.

Proof. Let $\Pi$ be the product of the first $n$ primes and let $k$ be $\Pi$ divided by $p$. Then $k \in K_{n}$, so $k \in R(\sigma)$. Plainly, $q$ divides $k$ and $p$ does not. Lemma 3.2 now implies the conclusion that $S_{p}$ does not include $S_{q}$.

Of course, Lemmas 3.1 and 3.3 imply that the dimension of $K_{n}$ is no less than $\log _{2}(n+1)$, so $K$ does not possess a finite dimension.

4. Permutable products and conditional independence. Let $G$ be a topological Hausdorff group which is faithfully represented as a group of measure preserving transformations of a countably additive probability space $(\Omega, \mathscr{F}, P)$ for which the mapping $(g, \omega) \rightarrow g(\omega)$ is jointly measurable. For each compact subgroup $H$ of $G$, let $E_{H}$ be the operator

$$
\left(E_{H} X\right)(\omega)=\int_{H} X(h(\omega)) d h,
$$

where $d h$ is normalized Haar measure on $H$. Of course, $E_{H}$ is well defined, at least on the space of all bounded, $\mathscr{F}$-measurable, random numbers $X$, and is a version of the conditional expectation operator associated with the $\sigma$-field of $H$-invariant, $\mathscr{F}$-measurable, subsets of $\Omega$.

The product $S T$ of subgroups $S$ and $T$ of $G$, is the set of all products $s t$ for $s \in S$ and $t \in T$, and $S \vee T$ is the smallest subgroup of $G$ which includes both $S$ and $T$. Of course, $S T \subset S \vee T$, and the reverse conclusion holds if, and only if, $S T=T S$, in which event $S$ and $T$ are permutable and $S T$ is the permutable product of $S$ and $T$, a terminology borrowed from finite group theorists. 
Proposition 4.1. Let $S$ and $T$ be compact subgroups of $G$. If $S$ and $T$ are permutable, then $E_{S}$ and $E_{T}$ commute. If $G=\Omega$ is compact, $G$ acts on $\Omega$ by left translation, and $P$ is normalized Haar measure on $G$, then the converse also holds.

Proof. As is easily verified, it suffices to give the proof in the special case-henceforth assumed- that $G$ is a compact group acting on itself by left translation. For this special case, (4.1) becomes:

$$
E_{H} u(g)=\int_{H} u(h g) d h,
$$

where $u$ ranges over the bounded, Borel functions defined on $G, g$ ranges over $G$ and $h$ over $H$.

Call $u T$-invariant if $u(t g)$ is $u(g)$ for all $t \in T$ and all $g \in G$.

LEMMA 4.1. If $S T=T S$ and $u$ is $T$-invariant and continuous, then

$$
\int_{H} u(h) d h=\int_{S} u(s) d s,
$$

where dh and ds are normalized Haar measures on $H=S T$ and on $S$.

Proof of (4.2). As von Neumann's theory of invariant integration ([12], [14]) makes evident when $G$ is second countable-and as is valid without this hypothesis-for each $\varepsilon>0$ there exists $s_{i} \in S, i=1, \ldots, n$, such that $(1 / n) \Sigma_{i} u\left(s s_{i}\right)$ differs from the right-hand side of (4.2), say $c$, by at most $\varepsilon$, uniformly in $s \in S$. Since $u$ is $T$-invariant, $(1 / n) \Sigma_{i} u\left(h s_{i}\right)$, therefore, differs from $c$ by at most $\varepsilon$, uniformly in $h \in T S=H$. But the only number $c$ which has this property is the left-hand side of (4.2), so the lemma is proved.

Continuation of Proof of Proposition 4.1. Suppose that $S$ and $T$ are compact and permutable. The program is to show that

$$
E_{S T} f=E_{S} E_{T} f
$$

for all bounded, Borel $f$, which plainly implies that $E_{S}$ and $E_{T}$ commute. Because the collection of $f$ which satisfy (4.3) is closed under uniformly bounded, pointwise convergence, it suffices to establish (4.3) for continuous $f$. Since $T \subset S T$, the left-hand side of (4.3) is $E_{S T} E_{T} f$, so it even suffices to show:

$$
E_{S T} E_{T} f=E_{S} E_{T} f
$$

for continuous $f$. Moreover, since $E_{T} f$ is plainly $T$-invariant, it is enough to establish

$$
E_{S T} v=E_{S} v
$$

for all $T$-invariant, continuous $v$. For $H=S T, h \in H, g \in G$, write $v_{g}(h)=$ $v(h g)$ and calculate, thus. 


$$
\begin{aligned}
E_{S T} v(g) & =\int_{H} v(h g) d h=\int_{H} v_{g}(h) d h \\
& =\int_{S} v_{g}(s) d s=\int_{S} v(s g) d s=E_{S} v(g),
\end{aligned}
$$

where the first equality is by definition of $E_{H}$, the second by definition of $v_{g}$, the third by Lemma 4.1 applied to $v_{g}$, the fourth by definition of $v_{g}$, and the last by definition of $E_{S}$. This proves (4.5), hence (4.3) and the first assertion of Proposition 4.1.

Consider now the converse. We thank Michael Cowling for suggesting the following demonstration, which is much simpler than our original one. For a bounded, continuous function $f$ on $G$ and a probability measure $\mu$, define a function $f^{*} \mu$ by

$$
f^{*} \mu(x)=\int_{G} f(x g) \mu(d g) .
$$

Then $E_{S} f=f^{*} \mu_{S}$ where $\mu_{S}$ is Haar measure on $S$. For the convolution $\mu^{*} \nu$ of two probability measures $\mu$ and $\nu$ defined in the usual way, $f^{*}\left(\mu^{*} \nu\right)=$ $\left(f^{*} \mu\right)^{*} \nu$. So $E_{S} E_{T}=E_{T} E_{S}$ implies that $\mu_{S}^{*} \mu_{T}=\mu_{T}^{*} \mu_{S}$. Since $S$ and $T$ are the respective supports of $\mu_{S}$ and $\mu_{T}$, and since the support of the convolution of two measures is the product of their supports, $S T=T S$, and the proof is complete.

Proposition 4.2. Let $G$ be a topological Hausdorff group of measure preserving transformations acting on a countably additive probability space $(\Omega, \mathcal{F}, P)$ and suppose that the action is jointly measurable. Suppose, too, that for each $i \in N^{d}, H(i)$ is a compact subgroup of $G$ and that $H(i \vee j)$ is the permutable product of $H(i)$ and $H(j)$ for all $i, j \in N$. Then for each $X \in \mathcal{L} \log ^{d-1} \mathcal{E}$, $\int X(g(\omega)) d_{i}(g)$ converges for P-almost all $\omega$ to $X_{\infty}(\omega)$ as $i \rightarrow \infty$, where $d_{i}(g)$ refers to normalized Haar measure on $H(i)$, and where $X_{\infty}$ is the conditional expectation of $X$ with respect to the $\sigma$-field of $\mathcal{F}$-events which are invariant under $\cup_{i} H(i)$. Moreover, if for each $1 \leqslant r \leqslant d$ the $\sigma$-field $\mathscr{F}_{r, \infty}$ is $P$-trivial,

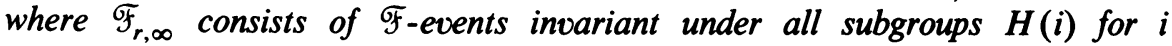
belonging to the rth axis then for $P$-almost all $\omega$, the convergence is rearrangeable and $X_{\infty}(\omega)=E(X)$.

Proof. Apply Proposition 4.1, the convergence theorem of Gut [1976] mentioned in $\$ 2$, and Theorem 4.2.

Let $Y=\left\{Y_{n, m}, n, m \in N\right\}$ be a two-parameter, real-valued stochastic process. Associated with $Y$ is the one-parameter, vector-valued, stochastic process $Y^{(1)}$, where, for each $n, Y_{n}^{(1)}$ is the row sequence $Y_{\mathrm{n}, \cdot}$. Also associated with $Y$ is the one-parameter, vector-valued, stochastic process $Y^{(2)}$ where, for each $m, Y_{m}^{(2)}$ is the column sequence $Y_{,, m}$. Call $Y$ semiexchangeable if $Y^{(1)}$ is an exchangeable, vector-valued, stochastic process, and $Y_{2}$ is, too. The notion 
that $Y$ is semiexchangeable can be introduced in an equivalent manner, thus. Let $H(n, 1)$ be the subgroup of permutations of $N^{2}$ consisting of the $n$ ! permutations which act by permuting the first $n$ columns and by leaving all other columns fixed. Let $H(1, m)$ be defined similarly in terms of rows. For $i=(n, m)$, let $H(i)$ be the product of $H(n, 1)$ and $H(1, m)$, which is a permutable product, and let $G$ be the union of $H(i)$ over $i \in N^{2}$. Let $\Omega=R^{N^{2}}, \mathscr{F}$ the usual $\sigma$-field on $\Omega$, that is the one generated by the evaluation mappings. Then $G$ acts in an obvious way on $\Omega$, and a process $Y$ is semiexchangeable if, and only if, the law of $Y$ on $\mathscr{F}$ is $G$-invariant. Of course, the notion of a stochastic process being semiexchangeable is easily extended to $d$-parameter, real-valued, stochastic processes $Y$. For such a process $Y=\left(Y_{i}, i \in N^{d}\right)$, define

$$
X_{i}=\sum_{j<i} Y_{j} /|i|
$$

where $|i|=\Pi_{r} i(r)$ is the number of $j$ with $j \leqslant i$.

COROLlaRy 4.1. If $Y=\left(Y_{i}, i \in N^{d}\right)$ is semiexchangeable and in

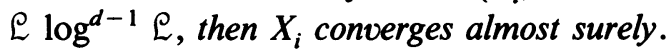

As a special case of a semiexchangeable process, let the $Y_{i}$ be i.i.d. The zero-one law of Hewitt-Savage [1955] then implies that each $\mathscr{F}_{r, \infty}$ is $P$-trivial, so Proposition 4.2 applies to yield the strong law of large numbers for multiparameter arrays due to Zygmund [1951], Smythe [1973]: If $E\left(\left|Y_{i}\right| \log ^{+}\left|Y_{i}\right|\right)^{d-1}<\infty$, then $X_{i}-E X_{i}$ converges rearrangeably almost surely to zero. The convergence theorem for generalized $U$-statistics of Sen [1977] could also be seen as a corollary to Proposition 4.2.

\section{REFERENCES}

1. R. C. Baker (1976), Riemann sums and Lebesgue integrals, Quart. J. Math. Oxford Ser. (2) 27, 191-198.

2. R. Cairoli (1970), Une inégalité pour martingales $\dot{a}$ indices multiples et ses applications, Seminaire de Probabilités IV, Lecture Notes in Math., vol. 124, Springer-Verlag, Berlin, pp. 1-28.

3, R. Cairoli and J. B. Walsh (1975), Stochastic integrals in the plane, Acta Math. 134, 111-183.

4. C. Dellacherie and P. A. Meyer (1975), Probabilités et potentiel, Hermann, Paris.

5. J. Dieudonné (1950), Sur un théorème de Jessen, Fund. Math. 37, 242-248.

6. Lester E. Dubins (1977), Measurable, tail disintegrations of the Haar integral are purely finitely additive, Proc. Amer. Math. Soc. 62, 34-36.

7. N. Dunford (1951), An individual ergodic theorem for non-commutative transformations, Acta Sci. Math. (Szeged) 14, 1-4.

8. R. F. Gundy and N. Th. Varopoulos (1975), A martingale that occurs in harmonic analysis, Note No. 157, Analyse Harmonique d'Orsay, Université Paris XI.

9. A. Gut (1976), Convergence of reversed martingales with multidimensional indices, Duke Math. J. 43, 269-275.

10. E. Hewitt and L. J. Savage (1955), Symmetric measures on Cartesian products, Trans. Amer. Math. Soc. 80, 470-501. 
11. B. Jessen (1934), On the approximation of Lebesgue integrals by Riemann sums, Ann. of Math. (2) 35, 248-251.

12. J. von Neumann (1934), Zum Haarschen mass in Topologischon Grupp., Comput. Math. 1. 13. Jacques Neveu (1975), Discrete-parameter martingales, North-Holland, Amsterdam; American Elsevier, New York.

14. L. Pontriagin (1946), Topological groups, Princeton Univ. Press, Princeton, N.J.

15. Walter Rudin (1964), An arithmetic property of Riemann sums, Proc. Amer. Math. Soc. 15, 321-324.

16. P. K. Sen (1977), Almast sure convergence of generalized U-statistics, Ann. Probability 5, 287-290.

17. R. T. Smythe (1973), Strong laws of large numbers for r-dimensional arrays of random variables, Ann. Probability 1, 164-170.

18. (1974), Sums of independent random variables on partially ordered sets, Ann. Probability 2, 5, 906-917.

19. A. Zygmund (1951), An individual ergodic theorem for non-commutative transformations, Acta Sci. Math. (Szeged) 14, 103-110.

Department of Mathematics, University of California, Berkelley, Californin 94720 